\title{
Discrete Trigonometric Transform Thermography
}

\author{
by Ivan LASSO-MARTINEZ*, Humberto LOAIZA-CORREA* and Andres RESTREPO-GIRON*
}

\begin{abstract}
*Universidad del Valle, Programa de Posgrados en Ingeniería Eléctrica y Electrónica, Cali, Colombia,
\end{abstract} \{ivan.lasso, humberto.loaiza, andres.david.restrepo\}@correounivalle.edu.co

\section{Extended Abstract}

This paper presents a signal processing algorithm as a solution to the problem of detection and characterization of subsurface defects in structures, which uses pulsed thermography. The main algorithms that have also approached this problem are thermographic signal reconstruction (TSR), pulse phase thermography (PPT), principal component thermography (PCT), differential absolute contrast (DAC), high-order statistics (HOS), among others. However, none of these algorithms has focused on use of the principle of conservation of energy between time and frequency domains. This is the orientation given to the presented algorithm. Due to discrete trigonometric transforms (DTTs) properties, it was decided to use some of these transforms as a mathematical tool to carry out the previous approach. For this, choice was made between discrete cosine transforms (DCTs) and discrete sine transforms (DSTs). Because there are eight DCTs and eight DSTs, it was necessary to limit scope of this work to the evaluation of only two types among sixteen possible transforms: the DCT-2 and the DST-4 were chosen; the first for its great capacity for energy compaction. The other types will be evaluated in future work. The results lead to conclusion that the cumulative percentage of energy function, calculated with DCT-2 and DST-4, presents advantages similar to those of the phase signal used in the PPT; for example, it is not affected by non-uniform heating and allows quantifying the depth of defects.

Regarding the materials, nine synthetic thermogram sequences were used, designed and generated with ThermoCalc-6L TM that emulate physical characteristics of a CFRP flat surface with $2 \mathrm{~mm}$ thick. All sequences contain five defects located at different positions of the specimen. The locations of these defects do not change from one sequence to another. All defects of a sequence are at the same depth; however, depth increases from sequence to sequence. Table 1 lists depths at which the defects of each sequence are found. All defects have a thickness equal to $0.1 \mathrm{~mm}$.

Table 1. Defect depths in each sequence of thermograms

\begin{tabular}{|l|l|l|l|l|l|l|l|l|l|}
\hline Sequence of thermal images & 1 & 2 & 3 & 4 & 5 & 6 & 7 & 8 & 9 \\
\hline Depth of all defects $(\mathrm{mm})$ & 0.2 & 0.4 & 0.6 & 0.8 & 1.0 & 1.2 & 1.4 & 1.6 & 1.8 \\
\hline
\end{tabular}

Regarding the methods, the discrete transforms DCT-2, Eq. (1), and DST-4, Eq. (2), were used to map temperature profiles from time domain to frequency domain. For these two direct equations their unitary and unidimensional variants were chosen.

$$
\begin{gathered}
X^{c 2}(k)=p(k) \sum_{n=0}^{N-1} x(n) \cos \left(\frac{\pi}{N}\left(n+\frac{1}{2}\right) k\right), \quad p(k)=\sqrt{\frac{2-\delta(k)}{N}}, \quad k=0, \quad 1, \quad 2, \quad \cdots \quad N-1 \\
X^{s 4}(k)=p \sum_{n=0}^{N-1} x(n) \sin \left(\frac{\pi}{N}\left(n+\frac{1}{2}\right)\left(k+\frac{1}{2}\right)\right), \quad p=\sqrt{\frac{2}{N}}, \quad k=0, \quad 1, \quad 2, \quad \cdots \quad N-1
\end{gathered}
$$

where,

- $x(n)$ is discrete time sequence of samples acquired from temperature signal (the thermal profile).

- $\quad N$ is length of the sequence $x(n)$ (number of samples of the thermal profile, i.e., the number of thermograms).

- $n$ is index of samples (in time domain) of the sequence $x(n)$.

- $k$ is index of coefficients (in frequency domain) of the transformed sequence $X(k)$.

- $X^{c 2}(k)$ is the transformed sequence obtained by applying the DCT-2 to $x(n)$.

- $X^{s 4}(k)$ is the transformed sequence obtained by applying the DST-4 to $x(n)$.

- $\delta(k)$ is the unit impulse function, which is defined as $\delta(k=0)=1$ and $\delta(k \neq 0)=0$.

In order to explain the algorithm, the following mathematical notation is introduced. For a sequence acquired by pulsed thermography, each thermogram has $R$ rows and $C$ columns of pixels. Therefore, any pixel in that sequence must be contained between the intervals $0 \leq r \leq R-1,0 \leq c \leq C-1$ and $0 \leq n \leq N-1$; so, the sequence can be denoted as $x(r, c, n)$. However, to emphasize that the algorithm is applied in time dimension, the notation $x_{r, c}(n)$ was preferred; that is, for a pixel located in the coordinates $(r, c)$ variation of its temperature over time (the thermal profile) was analysed. 
Step 1 of algorithm. The DTT of the corresponding experiment is applied to each thermal profile. For example, for the second experiment, with Eq. (2), each thermal profile was mapped to frequency domain using DST-4, Eq. (3).

$$
x_{r, c}(n) \stackrel{D S T-4}{\longleftrightarrow} X_{r, c}^{s 4}(k)
$$

Step 2. For each sequence $X_{r, c}^{s 4}(k)$ the energy density spectrum $S(k)$ is calculated. This is done by squaring value of each coefficient, Eq. (4).

$$
S(k)=\left|X_{r, c}^{s 4}(k)\right|^{2}
$$

Step 3. Because DST-4 is an orthogonal and unitary transformation, that is orthonormal, then it satisfies the principle of energy conservation between time and frequency domains. Therefore, DST-4 fulfils the Parseval's theorem in the form of Eq. (5).

$$
E_{x_{r, c}}=\sum_{n=0}^{N-1}\left|x_{r, c}(n)\right|^{2}=\sum_{k=0}^{N-1}\left|X_{r, c}^{s 4}(k)\right|^{2}
$$

where, $E$ is the total energy of thermal profile $x(n)$ located in coordinates $(r, c)$. This means that total energy $E$ is $100 \%$ of energy registered in $(r, c)$. Therefore, percentage of energy from each coefficient of the sequence $X_{r, c}^{s 4}(k)$ is calculated as a function of its index $k$, Eq. (6).

$$
\text { percentage of energy }(k)=\frac{100}{E_{x_{r, c}}}\left|X_{r, c}^{s 4}(k)\right|^{2}
$$

Step 4. Cumulative percentage of energy (CPE) is calculated, Eq. (7), from the first coefficient $(k=0)$ to the last one $(k=N-1)$.

$$
C P E\left(k^{\prime}\right)=\sum_{k=0}^{k^{\prime}} \frac{100}{E_{x_{r, c}}}\left|X_{r, c}^{s 4}(k)\right|^{2}=\frac{100}{E_{x_{r, c}}} \sum_{k=0}^{k^{\prime}}\left|X_{r, c}^{s 4}(k)\right|^{2}, \quad 0 \leq k^{\prime} \leq N-1
$$

The main results applying these steps are summarized in the following figures. Figure 1 shows 10 coordinates $(r, c)$ chosen arbitrarily: 5 on the defects and 5 on a non-defective area. Non-uniform heating was present in all thermogram sequences (table 1) used in both experiments. In figure 2 two curves are observed but in fact there are ten. In the upper part there are five overlapping curves (red colour), which were obtained from thermal profiles of the coordinates p1, p2, p3, p4 and p5 on defects; and at the bottom are the other five, also superposed (blue colour), of the coordinates p6, p7, p8, p9 and p10 on non-defective area.

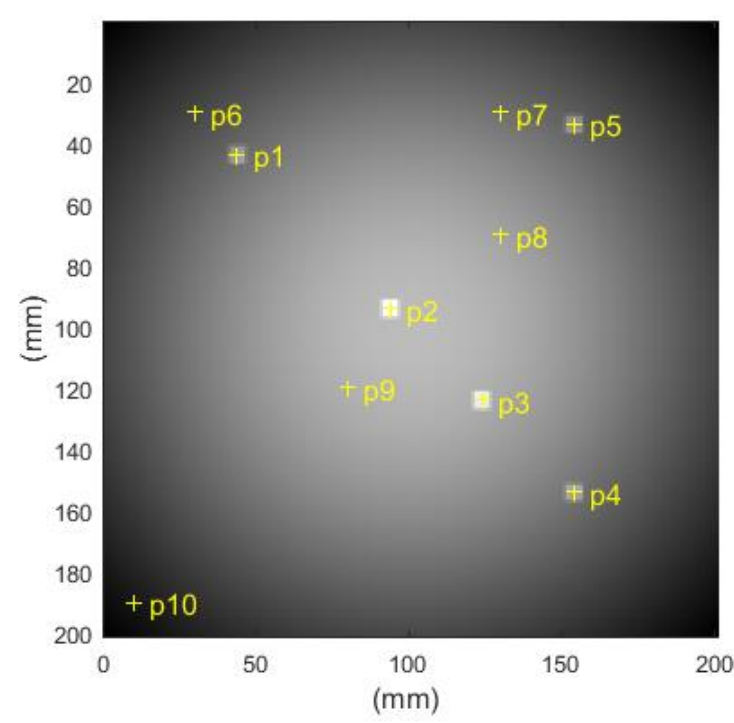

Fig. 1. Coordinates $(r, c)$

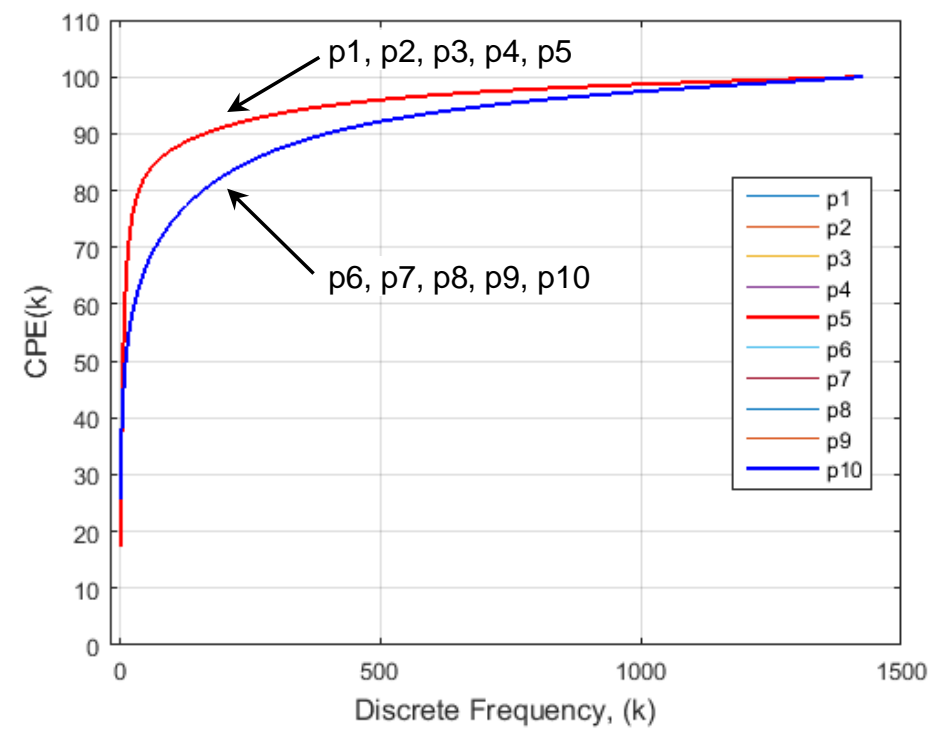

Fig. 2. Cumulative percentage of energy

The analysis of these results leads us to conclude that for the set of coordinates $(r, c)$ that belong to the region without defects (sound area) the same function $C P E(k)$ (blue) is obtained, which is practically unaffected by non-uniform heating. This also occurs with the coordinates of the defects; if these are at same depth, then have equal $C P E(k)$ (red). The normalization to 1 on $\mathrm{x}$ and $\mathrm{y}$ axes of the $C P E(k)$ functions make that these can be interpreted as Lorenz curves and used for detection of defects by means of the Gini index. The lowest index corresponds to the region without defects. 